\title{
Risk Indicators for Periodontitis in US Adults: NHANES 2009 to 2012
}

Paul I. Eke, ${ }^{*}$ Liang Wei, ${ }^{\dagger}$ Gina O. Thornton-Evans, ${ }^{\ddagger}$ Luisa N. Borrell, $§$ Wenche S. Borgnakke, Bruce Dye, $₫$ and Robert J. Genco\#

Background: Through the use of optimal surveillance measures and standard case definitions, it is now possible to more accurately determine population-average risk profiles for severe (SP) and non-severe periodontitis (NSP) in adults (aged 30 years and older) in the United States.

Methods: Data from the 2009 to 2012 National Health and Nutrition Examination Survey were used, which, for the first time, used the "gold standard" full-mouth periodontitis surveillance protocol to classify severity of periodontitis following suggested Centers for Disease Control/American Academy of Periodontology case definitions. Probabilities of periodontitis by: 1) sociodemographics, 2) behavioral factors, and 3) comorbid conditions were assessed using prevalence ratios (PRs) estimated by predicted marginal probability from multivariable generalized logistic regression models. Analyses were further stratified by sex for each classification of periodontitis.

Results: Likelihood of total periodontitis (TP) increased with age for overall and NSP relative to nonperiodontitis. Compared with non-Hispanic whites, TP was more likely in Hispanics (adjusted [a]PR = 1.38; $95 \%$ confidence interval 95\% CI: 1.26 to 1.52 ) and non-Hispanic blacks (aPR = 1.35; 95\% CI: 1.22 to 1.50 ), whereas SP was most likely in non-Hispanic blacks (aPR $=1.82 ; 95 \% \mathrm{CI}: 1.44$ to 2.31 ). There was at least a 50\% greater likelihood of TP in current smokers compared with non-smokers. In males, likelihood of TP in adults aged 65 years and older was greater (aPR $=2.07 ; 95 \% \mathrm{CI}: 1.76$ to 2.43 ) than adults aged 30 to 44 years. This probability was even greater in women $(\mathrm{aPR}=3.15 ; 95 \%$ CI: 2.63 to 3.77). Likelihood of TP was higher in current smokers relative to non-smokers regardless of sex and periodontitis classification. TP was more likely in men with uncontrolled diabetes mellitus (DM) compared with adults without DM.

Conclusions: Assessment of risk profiles for periodontitis in adults in the United States based on gold standard periodontal measures show important differences by severity of disease and sex. Cigarette smoking, specifically current smoking, remains an important modifiable risk for all levels of periodontitis severity. Higher likelihood of TP in older adults and in males with uncontrolled DM is noteworthy. These findings could improve identification of target populations for effective public health interventions to improve periodontal health of adults in the United States. J Periodontol 2016;87:1174-1185.

\section{KEY WORDS}

Diabetes mellitus; dental health surveys; epidemiology; periodontitis; population surveillance; smoking.

* Division of Population Health, Centers for Disease Control and Prevention, Atlanta, GA.

$\dagger$ DB Consulting Group, Atlanta, GA.

$\neq$ Division of Oral Health, Centers for Disease Control and Prevention.

\& Department of Epidemiology and Biostatistics, Graduate School of Public Health and Health Policy, City University of New York, New York, NY.

Department of Periodontics and Oral Medicine, University of Michigan School of Dentistry, Ann Arbor, MI.

II National Institute of Dental and Craniofacial Research, Bethesda, MD.

\# UB Microbiome Center, Schools of Dental Medicine and Medicine and Biomedical Sciences, State University of New York at Buffalo, Buffalo, NY. 
$\mathrm{P}$ eriodontitis is a chronic disease of hard and soft tissues supporting teeth. ${ }^{1}$ Monitoring and reducing periodontitis in the adult population of the United States through national disease surveillance and public health interventions is a significant concern highlighted in the Surgeon General's Report on Oral Health, ${ }^{2}$ and in Healthy People 2020. ${ }^{3,4}$ To address this concern, it is crucial to better understand population characteristics, including modifiable and non-modifiable risk factors, associated with periodontitis in US adults.

Recent advances in surveillance of periodontitis in the United States, including recent changes in clinical examination protocols and development and use of standard case definitions, have improved understanding of the burden of periodontitis in the adult population. ${ }^{5,6}$ Historically, representative population estimates of periodontitis for US adults have been based on data from the National Health and Nutrition Examination Survey (NHANES). Prior to 2009, surveillance of periodontitis in NHANES was based on data collected by various Partial Mouth Periodontal Examination (PMPE) protocols. ${ }^{7,8}$ Identification of periodontitis cases based on PMPE measurements can result in false negatives, impacting accuracy of prevalence estimates and risk assessments due to misclassification bias. ${ }^{9}$ Using the Full Mouth Periodontal Examination (FMPE) protocol optimizes measurements for the most accurate classification of periodontitis. Since 2009, NHANES has used the FMPE protocol. Similarly, standardized case definitions of periodontitis are critical to proper characterization of true disease burden in populations, and for comparison among studies. In 2007, an expert workgroup developed and suggested standard case definitions for surveillance of periodontitis. ${ }^{5,6}$ Using data from the 2009 to 2012 NHANES surveys and applying these case definitions, the authors have revised prevalence of periodontitis in US adults, reporting for the first time (to the best knowledge of the authors) direct evidence that almost $50 \%$ of US adults aged 30 years and older have periodontitis. ${ }^{10}$

Availability of periodontal measures from an FMPE protocol and simultaneous collection of information on putative risk factors such as: 1) sociodemographic, 2) behavioral, and 3) comorbid conditions in the 2009 to 2012 NHANES surveys for the first time affords opportunity to revisit and determine more valid population-average risk profiles for periodontitis in US adults. Two studies have previously reported putative population risk factors for periodontitis, notably: 1) sociodemographic characteristics such as age, sex, race/ethnicity, and income; 2) risk behavior such as smoking; and 3) comorbid conditions such as diabetes mellitus (DM) and obesity. ${ }^{11,12}$ However, these studies are subject to limitations such as using periodontitis cases determined from PMPE measures and study samples not generalizable to the adult US population. ${ }^{11-13}$ Moreover, these studies have often used different and inconsistent case definitions that did not capture severity of periodontitis. ${ }^{11-13}$

Identifying population risk factors is critical to developing effective preventative interventions of disease in populations. Thus, data from the 2009 to 2012 NHANESs were used, based on FMPE and classification of severity of periodontitis based on the suggested Centers for Disease Control/American Academy of Periodontology (CDC/AAP) case definitions for surveillance of periodontitis, to determine population-average risk profiles for periodontitis in US adults at least 30 years of age.

\section{MATERIALS AND METHODS}

This study used publically released NHANES 2009 to 2010 and 2011 to 2012 data. NHANES is a stratified, multistage probability sample of the civilian noninstitutionalized population in the 50 US states and the District of Columbia. Technical details of the survey, including sampling design, periodontal data collection protocols, and data availability can be accessed from the NHANES website. ${ }^{14}$ Additional information on oral health data collection and quality of data for the 2009 to 2010 survey are described elsewhere. ${ }^{15}$ Oral health data collection protocols for the NHANES surveys 2009 to 2012 were approved by the CDC, National Center for Health Statistics Research Ethics Review Board, Atlanta, GA (equivalent to Institutional Review Boards), and all survey participants provided written informed consent.

In participants 30 years and older, periodontal examinations were conducted in a mobile examination center (MEC). Gingival recession (GR: distance between the free gingival margin [FGM] and cemento-enamel junction [CEJ]), followed by periodontal probing depth (PD) (distance from FGM to bottom of the sulcus or periodontal pocket) were measured at six sites around each tooth (mesio-, mid-, and disto-buccal; mesio-, mid-, and disto-lingual) for all teeth, excluding third molars. For measurements at each site, a periodontal probe with 2-4-6-8-10$12-\mathrm{mm}$ graduations was positioned parallel to the long axis of the tooth at each site. Each measurement was rounded to the lower whole millimeter. Data were recorded directly into a NHANES oral health data management program that instantly calculated attachment loss (AL) as PD minus GR. Adults aged 30 years or older, who had one or more natural teeth and did not have a health condition that required antibiotic prophylaxis before periodontal probing, were eligible for periodontal examination during NHANES 2009 to 2012. 
Periodontitis cases were defined following suggested CDC/AAP case definitions for surveillance of periodontitis. ${ }^{5,6}$ Severe periodontitis (SP) was defined as having two or more interproximal sites with $\geq 6 \mathrm{~mm} \mathrm{AL}$ (not on the same tooth) and one or more interproximal site(s) with $\geq 5 \mathrm{~mm}$ PD. Nonsevere periodontitis (NSP) combined two levels of disease: 1) moderate periodontitis, defined as two or more interproximal sites with $\geq 4 \mathrm{~mm} \mathrm{AL}$ (not on the same tooth) or two or more interproximal sites with PD $\geq 5 \mathrm{~mm}$ (not on the same tooth); and 2) mild periodontitis, defined as two or more interproximal sites with $\geq 3 \mathrm{~mm} \mathrm{AL}$ and two or more interproximal sites with $\geq 4 \mathrm{~mm}$ PD (not on the same tooth), or one interproximal site with $\geq 5 \mathrm{~mm}$ PD. Both categories are not truly ordinal as the label suggests because many of the "moderate" cases had insufficient PD to qualify as "mild," and therefore, they were combined under the label "non-severe" periodontitis. ${ }^{16}$ Total periodontitis (TP) (reported here as periodontitis) was defined as presence of SP or NSP. Among the 7,066 respondents, 16 respondents had one tooth only and were classified as non-cases.

Consistent with previous studies, 7,12,17,18 reported potential sociodemographic, behavior, and comorbid risk indicators for periodontitis were selected for analyses. Age (categorized as 30 to 44 years, 45 to 54 years, 55 to 65 years, and $>65$ years) and sex (male/ female) were included in the analyses as collected by NHANES. Race/ethnicity was analyzed in four groups: 1) non-Hispanic whites; 2) non-Hispanic blacks; 3) Hispanic (i.e., anyone who self-identifies as "Hispanic" which is a combination of Mexican-Americans and other Hispanics, of which the majority self-identify as Mexican-Americans); and 4) other race/ethnicity, including multiracial. Education was classified as: 1) less than high school; 2) high school graduate or General Education Development high school equivalency test; and 3) greater than high school. Poverty status categories or percentage of poverty relative to federal poverty levels were derived from: 1) family income; 2) family size, and number of children in the family for families with two or fewer adults; and 3) on the ages of adults in the household. Poverty level was based on definitions originally developed by the Social Security Administration. ${ }^{19}$ Families or individuals with income below their appropriate thresholds are classified as below the federal poverty level (FPL). These thresholds are updated annually by the US Census Bureau. ${ }^{20}$ Marital status was self-reported: 1 ) married; 2) divorced or separated; 3) widowed; 4) living with a partner; and 5) single/never married.

Smoking status was constructed from responses to two questions: 1) Have you smoked at least 100 cigarettes in your life?; and 2) Do you now smoke cigarettes? Respondents who reported yes to both questions were categorized as current smokers, respondents who reported currently not smoking but having smoked more than 100 cigarettes in the past were categorized as former smokers, and respondents who reported no to both questions were categorized as non-smokers.

DM status was defined by self-report, levels of fasting plasma glucose (FPG), or blood levels of glycosylated hemoglobin A1c (HbA1c or A1c). Participants who responded "Yes" to the question, "Have you ever been told by your doctor or other care provider that you had DM?" were considered to have diagnosed DM, and those who answered "No" were classified as not diagnosed. Uncontrolled DM was defined as diagnosed DM and FPG $\geq 126 \mathrm{mg} / \mathrm{dL}$ or $\mathrm{HbA}_{1 \mathrm{c}} \geq 7.0$; controlled DM was defined as diagnosed DM and FPG $<126 \mathrm{mg} / \mathrm{dL}$ or HbA $1 \mathrm{c}<7.0$; preDM was defined as no diagnosed DM and $100 \leq F P G \leq 126 \mathrm{mg} / \mathrm{dL}$ or $5.7 \leq \mathrm{HbA} 1 \mathrm{c} \leq 6.5$; and non-DM was defined as selfreported no diagnosed DM and FPG $<100 \mathrm{mg} / \mathrm{dL}$ or $\mathrm{HbA}_{1 \mathrm{c}}<5.7$. Undiagnosed DM was defined as no diagnosed DM and FPG $\geq 126$ or HbA1c $\geq 6.5 .21,22 \mathrm{Du}$ ration of DM was categorized as 0 to 2, 3 to 6,7 to 10,11 to 17 , and $\geq 18$ years, since being diagnosed by a doctor as having DM. Body mass index (BMI) was used to determine levels of obesity status. An individual with BMI $\geq 30$ was considered obese, between 25 and 30 overweight, from 18.5 to $<25$ normal weight, and $<18.5$ as underweight. ${ }^{23,24}$

In NHANES 2009 to 2012, adults aged 30 years or older with at least one natural tooth and not suffering from any health condition requiring antibiotic prophylaxis prior to periodontal probing were eligible for periodontal examination. Of 9,402 adults aged 30 years and older who participated in the survey, 1,631 were excluded from the oral health assessment in the MEC due to medical exclusions or not completing their examination, and 705 were identified as edentulous (edentate, having no teeth). Analyses were based on the remaining 7,066 participants $(3,515$ males and 3,551 females) representing a weighted population of approximately 141.0 million civilian non-institutionalized American adults 30 years of age and older. When calculating prevalence ratio for NSP, SP cases were excluded from the denominator, whereas NSP cases remained in the denominator when estimating the prevalence ratio for SP. Sample sizes varied according to outcome definitions and covariates included in regressions models. (Sample size for each model is provided in the respective table footnotes.)

Descriptive statistics were calculated by sex and severity of periodontitis (i.e., SP, NSP, and TP). Accounting for high prevalence of TP $(>10 \%)$ in the US adult population, prevalence ratios (PRs) were used to avoid overestimation of association between 


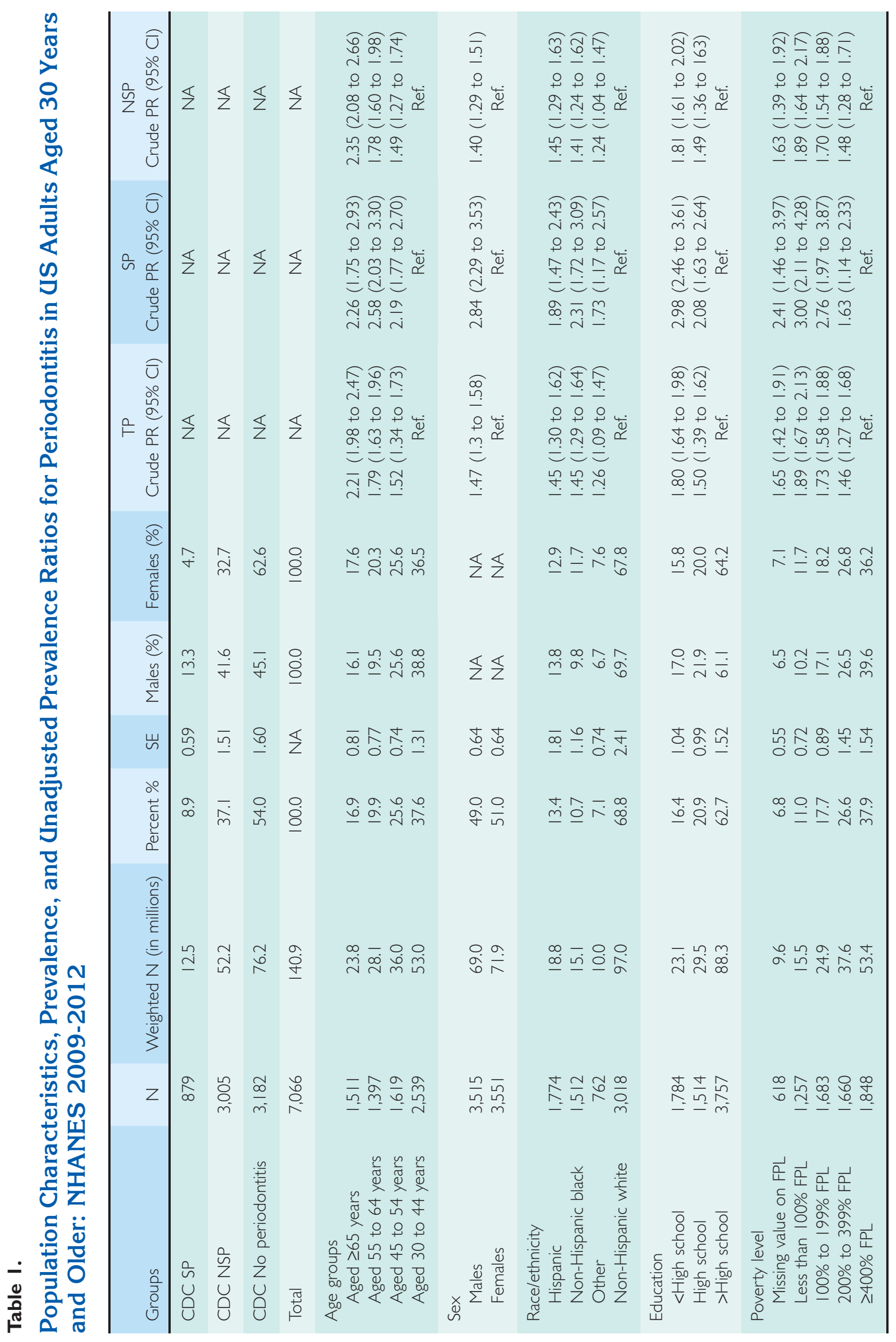




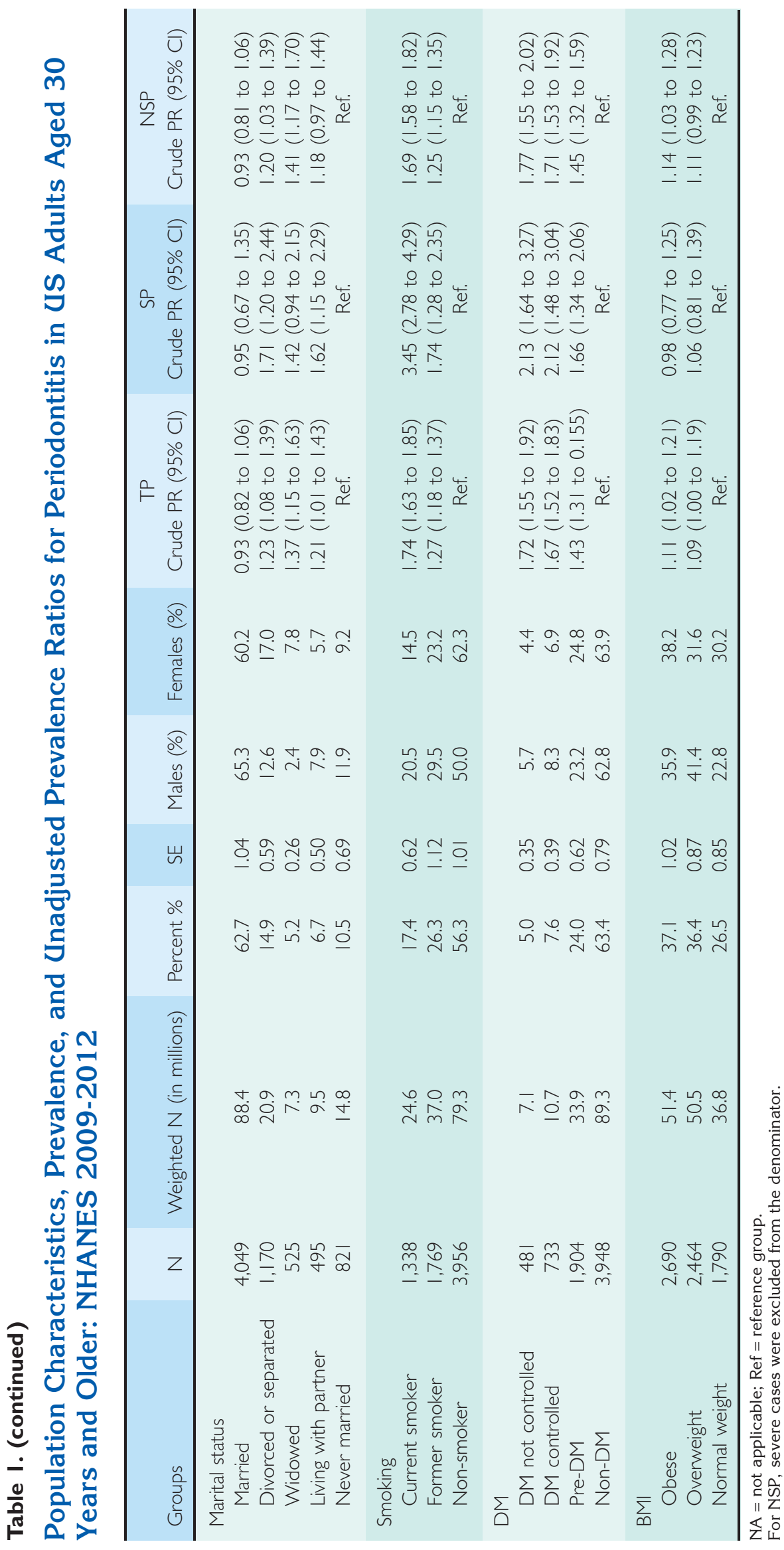




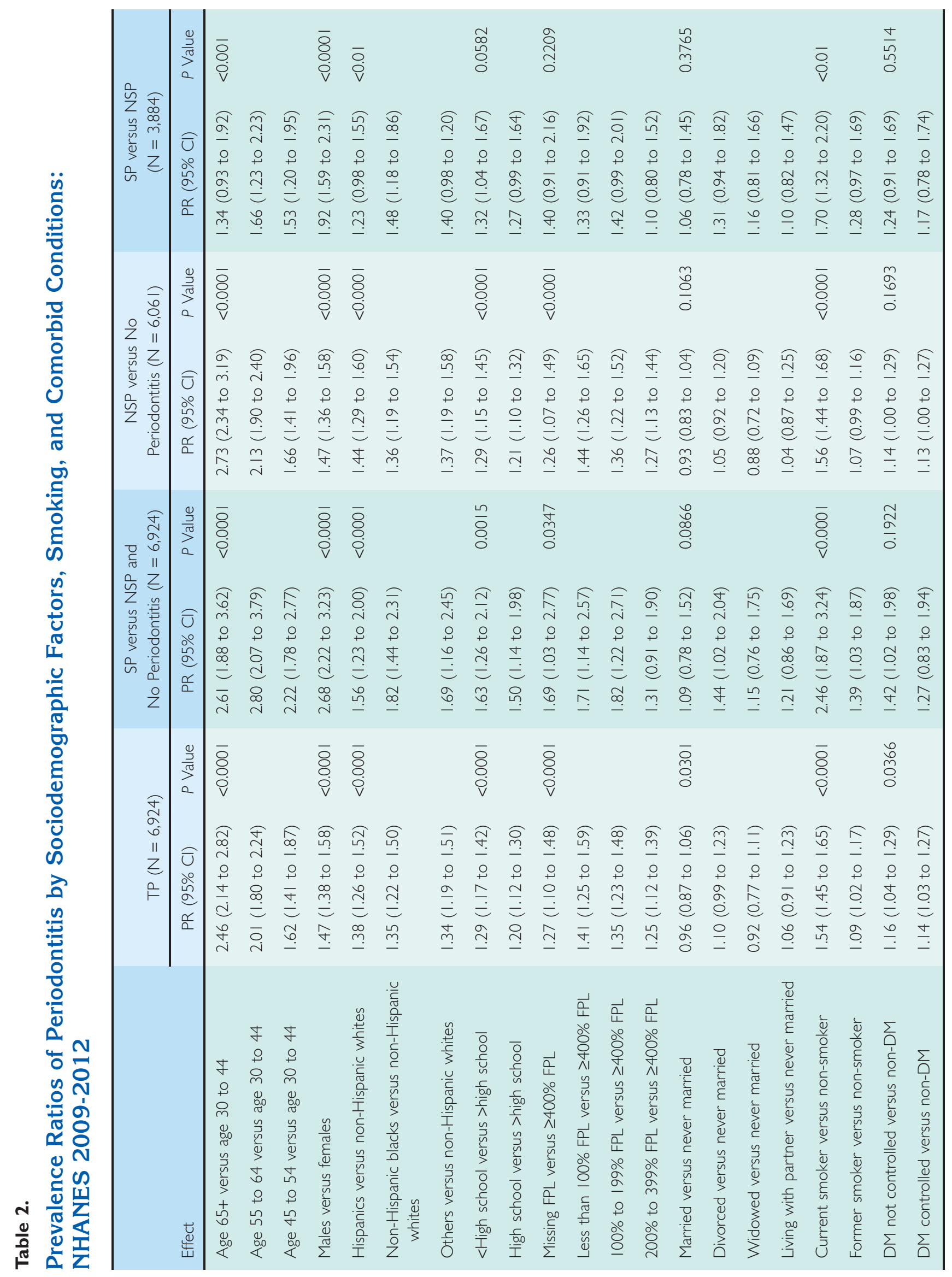




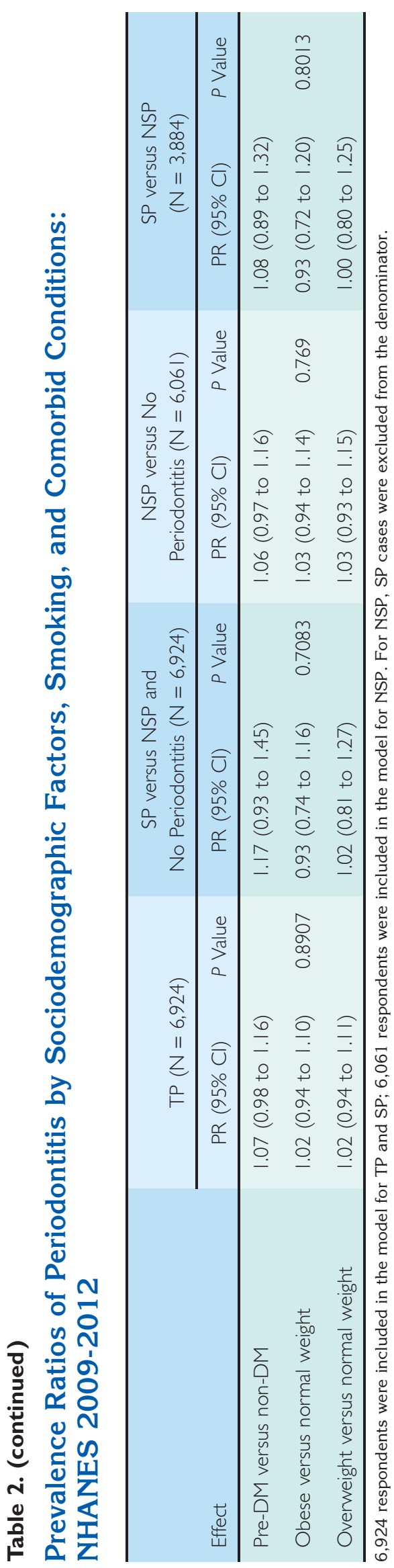

each exposure and periodontitis. Adjusted weighted predicted PRs and 95\% confidence interval (95\% CI) for likelihood of having periodontitis (TP, SP, or NSP) by selected covariates were calculated using logbinomial regression models. All analyses were conducted using callable add-on version of statistical analysis software ** and NHANES MEC examination weights to account for the complex survey sampling design.

\section{RESULTS}

Overall, a total of $46.0 \%$ US adults aged 30 and older had TP, distributed as $8.9 \%$ with SP and $37.1 \%$ with NSP. More males than females had SP $(13.3 \%$ versus $4.7 \%)$ and NSP (41.6\% versus $32.7 \%)$. The study population was fairly evenly distributed by sex $(51 \%$ females), but more males than females were current and former smokers, had DM, and were overweight (Table 1).

Using multivariable analyses (Table 2), likelihood of having TP or NSP increased steadily with increasing age, whereas this pattern was not observed for SP. Likelihood of TP double as high in males compared with females, with the highest probability observed for $\mathrm{SP}$ (adjusted prevalence ratio [aPR] $=2.68 ; 95 \% \mathrm{CI}$ : 2.22 to 3.23). TP was most likely in Hispanics (aPR = 1.38; $95 \%$ CI: 1.26 to 1.52 ) and non-Hispanic blacks ( $\mathrm{aPR}=1.35 ; 95 \% \mathrm{CI}: 1.22$ to 1.50$)$, and SP was more likely in non-Hispanic blacks ( $\mathrm{aPR}=1.82 ; 95 \% \mathrm{CI}$ : 1.44 to 2.31) compared with non-Hispanic whites. Adults with less than a high school education were more likely to have TP, with the highest probability observed for SP (aPR $=1.63 ; 95 \% \mathrm{CI}: 1.26$ to 2.12 ) and NSP (aPR=1.29; 95\% CI: 1.15 to 1.45 ) than those with greater than a high school education. Highest probability for SP was seen in adults with $100 \%$ to $199 \%$ of FPL (aPR $=1.82 ; 95 \% \mathrm{CI}: 1.22$ to 2.71 ), whereas highest probability for NSP was seen in the poorest at less than $100 \mathrm{FPL}$ (aPR $=1.44 ; 95 \% \mathrm{CI}$ : 1.26 to 1.56 ). Overall, there was a steady increase in likelihood of TP with increasing poverty (lower FPL). TP was significantly more likely in current and formers smokers compared with non-smokers. Likelihood for TP was highest in current smokers (aPR 1.54; 95\% CI: 1.45 to 1.65), and smoking was strongly associated with SP $(\mathrm{aPR}=2.46 ; 95 \% \mathrm{CI}: 1.87$ to 3.24$)$. In participants with DM, TP was more likely in those with uncontrolled DM, specifically in those with SP (aPR $=1.42 ; 95 \% \mathrm{CI}: 1.02$ to 1.98). TP was not significantly associated with obesity status. Finally, likelihood of SP was highest in adults aged $>65$ years, in males, non-Hispanic blacks, and current smokers when compared with those with NSP. 


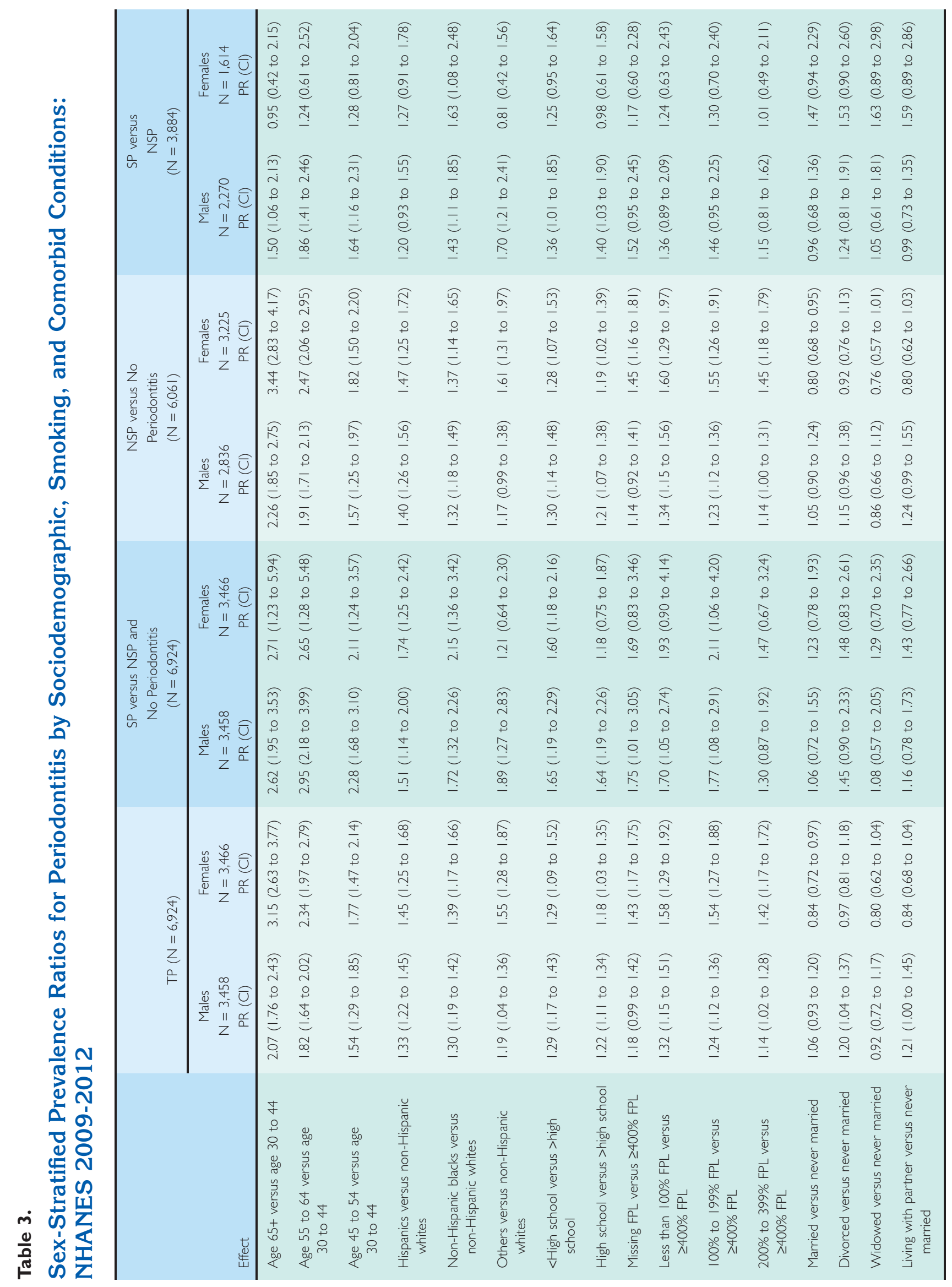




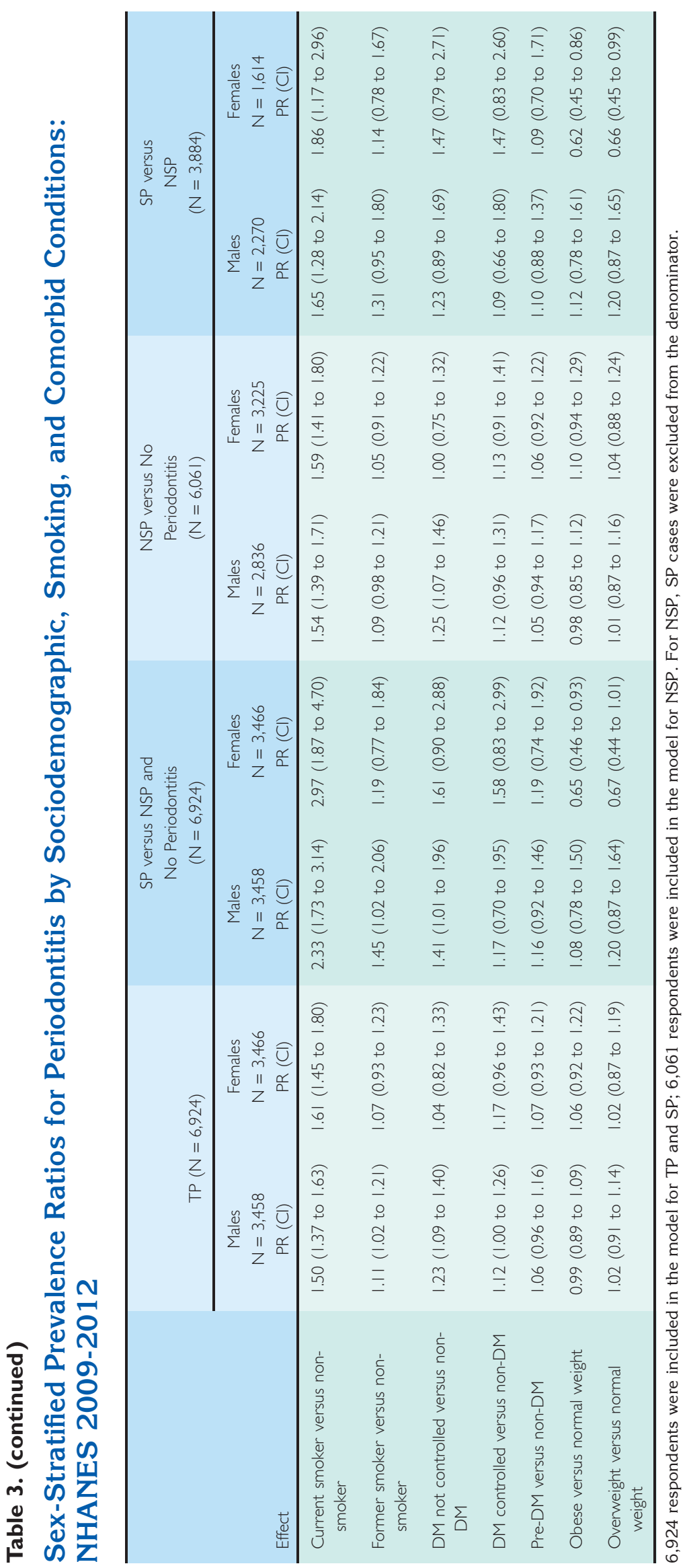

When stratified by sex and compared to the 30- to 44-year olds (Table 3, Fig. 1), TP and NSP (but not SP) were more likely in females 65 years and older. TP was equally likely in female former smokers and non-smokers. In females, TP and NSP were not significantly associated with DM status. In males, all levels of severity of periodontitis were more likely in participants with uncontrolled DM compared with participants without DM. Finally, when compared with participants with NSP, likelihood of SP was significantly higher in males of all age groups and education status, and for non-Hispanic blacks and current smokers regardless of sex.

Further analyses of TP and DM also suggested duration of DM did not significantly correlate with likelihood of TP after adjusting for all covariates (Wald $\mathrm{F}$ test, $P>0.05)$. Similarly, no trend was observed between TP and fasting glucose in participants with DM (Wald $F$ test, $P>0.05$ ). In females without DM, TP and NSP (but not SP) significantly increased with increasing levels of fasting glucose levels $(P<0.05)$. In males without DM, no trend was observed for any level of severity of periodontitis and increasing fasting glucose levels $(P>0.05)$. TP was significantly associated with increasing levels of $\mathrm{HbA} 1 \mathrm{c}$ in both males and females without DM $(P<0.05)$. In participants with DM, only SP was significantly correlated with increasing levels of HbA1c in males $(P>0.05$, results not shown).

\section{DISCUSSION}

This study finds new and important risk profiles for TP by severity of periodontitis and sex. Notably, differences are reported in likelihood of NSP and SP by increasing age, and a greater likelihood of TP in older adults ( $\geq 65$ years), with this likelihood being greater in females. While the probability of periodontitis was consistently associated with current smoking, it was found periodontitis was only significant in male former smokers but not in females. Periodontitis was not more likely in persons with controlled DM, only more likely in males with uncontrolled DM. Finally, this study did not find that periodontitis was more likely associated with obesity status, regardless of sex.

In this study, a higher likelihood of TP in older women compared with males aged 

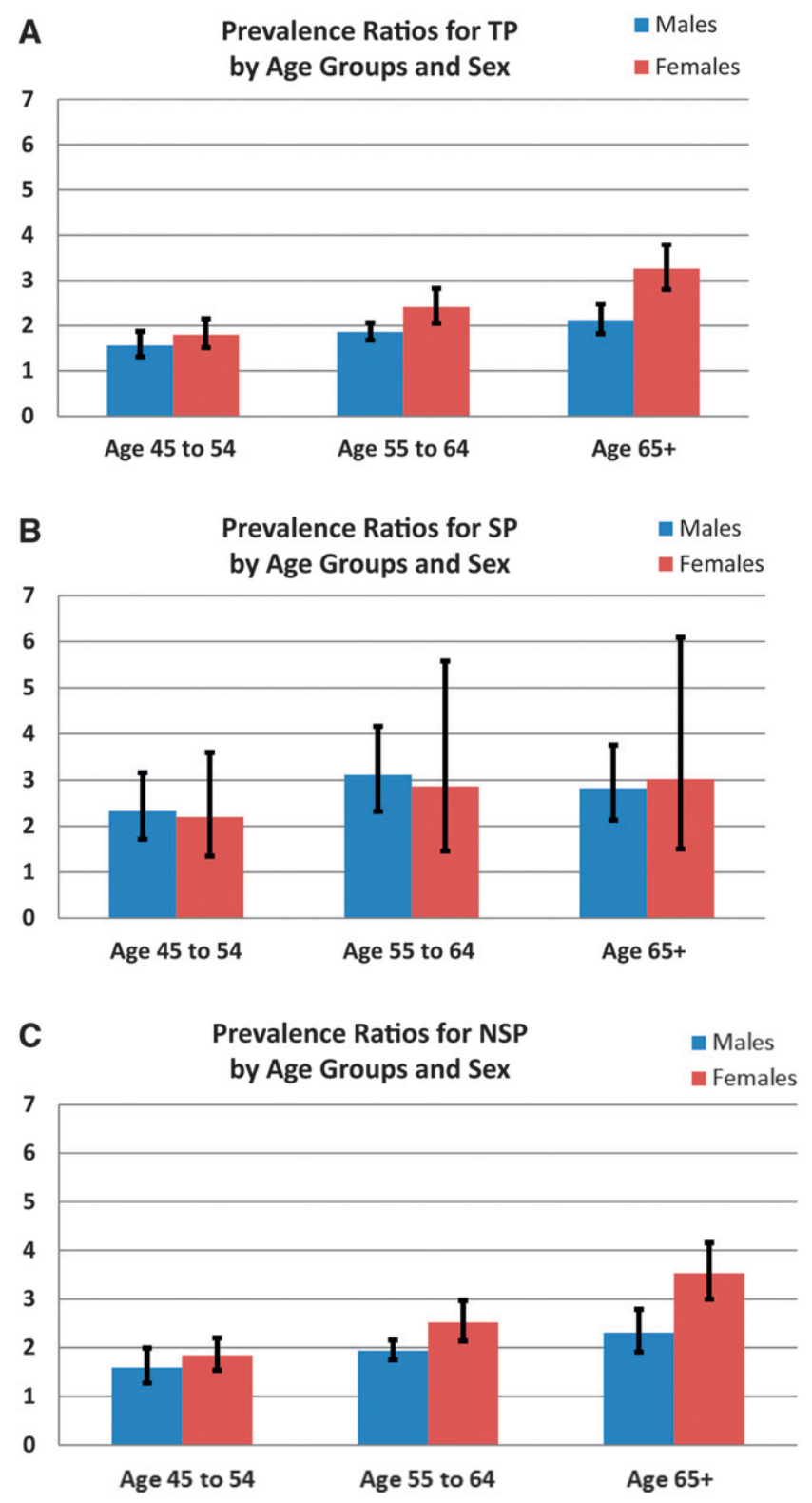

Figure I.

Prevalence ratios by age groups and sex: $\boldsymbol{A})$ TP; $\boldsymbol{B})$ SP; and $\mathbf{C})$ NSP (mild or moderate).

$\geq 65$ years was identified. This finding is not widely recognized in periodontal epidemiology even though a similar national study in the United States also reported that older men had better periodontal status than women. ${ }^{25}$ The higher likelihood of periodontitis in older women might be attributed to women keeping more of their teeth into older ages than men. Older women aged 65 years and more are also likely to be postmenopausal when production of estrogen has decreased. Lower estrogen production has been associated with increased risk for periodontitis. ${ }^{26}$ In the year 2000, approximately 45.6 million women in the United States were in the postmenopausal phase of life, and more women are living beyond the age of 65 years. ${ }^{20,27}$ Sexual dimorphism is known to exist for several chronic diseases and a recent study on classification of periodontitis based on gene expression demonstrated severity and extent of periodontitis are most strongly linked with sex compared with other putative risk factors for periodontitis. ${ }^{28}$ However, results from the current study could be due to a lower prevalence of disease in the reference group (women aged 30 to 44 versus men aged 30 to 44 ) and/or to a higher prevalence of disease in the women aged 65 and older.

Consistent with previous studies, ${ }^{25,29}$ it was found that likelihood of periodontitis is highest in current smokers. Likelihood of periodontitis dropped significantly in former smokers, and was not significantly different from non-smokers in females. This finding supports potential benefits of smoking cessation in preventing and controlling periodontitis. In 2013, an estimated one in five (17.8\%) of US adults were current smokers. ${ }^{30,31}$ Moreover, it is worth noting a higher, but non-significant, likelihood for periodontitis was reported in female current smokers compared with men. Postmenopausal female smokers have been reported to experience more severe alveolar bone loss than non-smokers, as smoking and osteoporosis/ osteopenia combine to exacerbate bone loss. ${ }^{32}$ However, findings from the current study could be due to a lower prevalence of disease in the reference group (female non-smokers versus male non-smokers) or due to a higher prevalence of disease in female current smokers.

Determination of DM status was based on results from actual laboratory tests of fasting plasma glucose and HbA1c, objective measures of DM status. Whereas relationships between periodontitis and DM have been widely reported in clinical studies, evidence for this association by severity of disease and sex at the population level is scarce. Findings from the current study are consistent with reports that SP is more prevalent in people with DM. Specifically, about one-third of people with DM have severe forms of periodontal disease, and adults aged 45 years or older, with poorly controlled DM, are three times more likely to have SP than those without DM. ${ }^{33,34}$ Also, a recent prospective population study similarly reports significant relationships between periodontitis and DM only in persons with uncontrolled DM. ${ }^{35}$

In the US population, $9.3 \%$ of adults have diagnosed (21.0 million) and undiagnosed ( 8.1 million) DM, with $12.3 \%$ of adults aged 20 and older having DM. ${ }^{36}$ Among the latter with diagnosed DM, about $86 \%$ use antidiabetic medication (insulin [14\%], oral medication [57\%], or both [15\%]), so only about $14 \%$ do not take medications to control their DM. ${ }^{36}$ This 
distribution may help explain findings of no significant probability of periodontitis associated with DM in general.

No increased likelihood of periodontitis associated with obesity status was reported, regardless of sex or severity of periodontitis. This finding is consistent with that from another population-based study. ${ }^{37}$ In contrast, a recent systemic review of five studies reported an association among weight gain and obesity and higher risk for new incident cases of periodontitis. Notably, US population studies included in that review used self-report periodontitis and a single measure of PD as the outcome. Importance of obesity to, and its influence on, periodontitis is attributed to BMI being a main risk factor for pre-DM. ${ }^{38}$ In this study, increased likelihood of periodontitis among participants with pre-DM was not found. While some studies have implicated obesity as a putative risk factor for periodontitis, most of these studies were conducted in hospital patients or in small samples. ${ }^{39,40}$

The current findings confirm that several socioeconomic indicators, such as: 1) race/ethnicity, 2 ) poverty level, and 3) education, are associated with increased probabilities for periodontitis, and therefore may account for disparities in periodontitis across the US adult population. For these indicators, however, effects were not found to be modified by sex. Borrell and Crawford $^{18}$ discussed how these socioeconomic variables interact to influence racial/ethnic disparities in populations. Particularly, they conclude periodontal disease is inversely related to education and income after controlling for selected characteristics, and differences in education and income explain most, if not all, of observed race and ethnicity disparities in periodontitis.

This study has some notable limitations. The study is cross-sectional, and therefore does not allow any deduction of causality or temporal relationship. The included population was limited to adults aged 30 years and older and did not include institutionalized persons, such as older adults in nursing home settings and adults in prisons, which may introduce some selection bias. There are possibilities of misclassified periodontitis cases because third molars were excluded, furcation involvement status and bleeding on probing (a parameter that could indicate active inflammation) were not assessed. Finally, a small number of non-severe cases may be misclassified as non-cases based on the classification criteria used.

\section{CONCLUSIONS}

Using optimal surveillance measures and standardized case definitions, this study provides new information on potential risk indicators for periodontitis that could guide public health action to prevent or attenuate periodontitis in the contemporary US adult population aged 30 years and older. Cigarette smoking, specifically current smoking, remains an important modifiable risk for all severity levels of periodontitis. The higher burden of periodontitis in older adults, especially women, and differences in potential risk profiles for SP and NSP by sex is noteworthy. Findings from the current study provide further information to assist in identifying segments of the population with higher probability for periodontitis, which might be used in developing targeted initiatives and programs to improve oral health, general health, and well-being of the adult US population.

\section{ACKNOWLEDGMENTS}

The authors acknowledge the contributions from the CDC Periodontal Disease Surveillance Workgroup. ${ }^{41}$ Dr. Wei is a contractor for the DB Consulting Group, Atlanta, Georgia. The authors report no conflicts of interest related to this study.

\section{REFERENCES}

1. Pihlstrom BL, Michalowicz BS, Johnson NW. Periodontal diseases. Lancet 2005;366:1809-1820.

2. U.S. Department of Health and Human Services. Oral health in America: A report of the Surgeon General. Rockville, MD: U.S. Department of Health and Human Services, National Institute of Dental and Craniofacial Research, National Institutes of Health; 2000:33-59. Available at: http://www.nidcr.nih.gov/DataStatistics/ SurgeonGeneral/Documents/hck1ocv.@www.surgeon. fullrpt.pdf. Accessed August 12, 2016.

3. U.S. Department of Health and Human Services. Healthy People 2020: Oral health. Available at: http:// www.healthypeople.gov/2020/topicsobjectives2020/ overview.aspx?topicid=32. Accessed August 12, 2016.

4. Eke PI, Thornton-Evans G, Dye B, Genco R. Advances in surveillance of periodontitis: The Centers for Disease Control and Prevention periodontal disease surveillance project. J Periodontol 2012;83:1337-1342.

5. Page RC, Eke PI. Case definitions for use in populationbased surveillance of periodontitis. J Periodontol 2007; 78(Suppl. 7):1387-1399.

6. Eke PI, Page RC, Wei L, Thornton-Evans G, Genco RJ. Update of the case definitions for population-based surveillance of periodontitis. J Periodontol 2012;83: 1449-1454.

7. Dye BA, Tan S, Smith V, et al. Trends in oral health status: United States, 1988-1994 and 1999-2004. National Center for Health Statistics. Vital Health Stat 2007:11(248).

8. Dye BA, Thornton-Evans G. A brief history of national surveillance efforts for periodontal disease in the United States. J Periodontol 2007;78(Suppl. 7): 1373-1379.

9. Eke PI, Thornton-Evans GO, Wei L, Borgnakke WS, Dye BA. Accuracy of NHANES periodontal examination protocols. J Dent Res 2010;89:1208-1213.

10. Eke PI, Dye BA, Wei L, Thornton-Evans GO, Genco RJ; CDC Periodontal Disease Surveillance workgroup: James Beck (University of North Carolina, Chapel Hill, USA), 
Gordon Douglass (Past President, American Academy of Periodontology), Roy Page (University of Washin. Prevalence of periodontitis in adults in the United States: 2009 and 2010. J Dent Res 2012;91:914-920.

11. Albandar JM. Epidemiology and risk factors of periodontal diseases. Dent Clin North Am 2005;49:517532, v-vi.

12. Genco RJ, Borgnakke WS. Risk factors for periodontal disease. Periodontol 2000 2013;62:59-94.

13. Beck JD, Offenbacher S. Systemic effects of periodontitis: Epidemiology of periodontal disease and cardiovascular disease. J Periodontol 2005;76(Suppl. 11): 2089-2100.

14. Centers for Disease Control and Prevention. National Health and Nutrition Examination Survey. Questionnaires, Datasets, and Related Documentation. Available at: http://www.cdc.gov/nchs/nhanes/nhanes_questionnaires. htm. Accessed August 12, 2016.

15. Dye BA, Li X, Lewis BG, Iafolla T, Beltran-Aguilar ED, Eke PI. Overview and quality assurance for the oral health component of the National Health and Nutrition Examination Survey (NHANES), 2009-2010. J Public Health Dent 2014;74:248-256.

16. Eke PI, Dye BA, Wei L, et al. Update on prevalence of periodontitis in adults in the United States: NHANES 2009 to 2012. J Periodontol 2015;86:611-622.

17. Borrell LN, Crawford ND. Social disparities in periodontitis among US adults: The effect of allostatic load. J Epidemiol Community Health 2011;65:144-149.

18. Borrell LN, Crawford ND. Socioeconomic position indicators and periodontitis: Examining the evidence. Periodontol 2000 2012;58:69-83.

19. Fisher GM. Poverty guidelines for 1992. Soc Secur Bull 1992;55:43-46.

20. US Department of Health \& Human Services, Office of the Assistant Secretary for Planning and Evaluation. The 2011 HHS poverty guidelines. Available at: https://aspe. hhs.gov/2011-hhs-poverty-guidelines. Accessed August 12, 2016.

21. American Diabetes Association. Diagnosis and classification of diabetes mellitus. Diabetes Care 2014;37 (Suppl. 1):S81-S90.

22. World Health Organization. Use of glycated hemoglobin (HbA1c) in the diagnosis of diabetes mellitus: Abbreviated report of a WHO consultation. Available at: http://www.who.int/diabetes/publications/reporthba1c_2011.pdf. Accessed August 12, 2016.

23. Keys A, Fidanza F, Karvonen MJ, Kimura N, Taylor HL. Indices of relative weight and obesity. $J$ Chronic Dis 1972;25:329-343.

24. Centers for Disease Control and Prevention. Healthy weight: About adult BMI. Available at: http://www.cdc. gov/healthyweight/assessing/bmi/adult_bmi/index. html. Accessed August 12, 2016.

25. Albandar JM. Global risk factors and risk indicators for periodontal diseases. Periodontol 2000 2002;29:177-206.

26. Friedlander AH. The physiology, medical management and oral implications of menopause. J Am Dent Assoc 2002;133:73-81.

27. USDHHS Administration on Aging. A Profile of Older Americans: 2000. Washington, DC: US Department of Health and Human Services, Administration on Aging; 2000.

28. Kebschull M, Demmer RT, Grün B, Guarnieri P, Pavlidis P, Papapanou PN. Gingival tissue transcriptomes iden- tify distinct periodontitis phenotypes. J Dent Res 2014 93:459-468.

29. Tomar SL, Asma S. Smoking-attributable periodontitis in the United States: Findings from NHANES III. National Health and Nutrition Examination Survey. $J$ Periodontol 2000;71:743-751.

30. Jamal A, Agaku IT, O'Connor E, King BA, Kenemer JB, Neff L. Current cigarette smoking among adults United States, 2005-2013. MMWR Morb Mortal Wkly Rep 2014;63:1108-1112.

31. Agaku IT, King BA, Dube SR; Centers for Disease Control and Prevention. Current cigarette smoking among adults - United States, 2005-2012. MMWR Morb Mortal Wkly Rep 2014;63:29-34.

32. Payne JB, Reinhardt RA, Nummikoski PV, Dunning DG, Patil KD. The association of cigarette smoking with alveolar bone loss in postmenopausal females. J Clin Periodontol 2000;27:658-664.

33. Saremi A, Nelson RG, Tulloch-Reid M, et al. Periodontal disease and mortality in type 2 diabetes. Diabetes Care 2005;28:27-32.

34. Taylor GW. Periodontal treatment and its effects on glycemic control: A review of the literature. Oral Surg Oral Med Oral Pathol Oral Radiol Endod 1999;87:311-316.

35. Demmer RT, Holtfreter B, Desvarieux $M$, et al. The influence of type 1 and type 2 diabetes on periodontal disease progression: Prospective results from the Study of Health in Pomerania (SHIP). Diabetes Care 2012;35: 2036-2042.

36. Centers for Disease Control and Prevention. National diabetes statistics report: Estimates of diabetes and its burden in the United States. Atlanta, GA: U.S. Department of Health and Human Services; 2014. Available at: http://www.cdc.gov/diabetes/pubs/statsreport14/ national-diabetes-report-web.pdf. Accessed August 12, 2016.

37. de Castilhos ED, Horta BL, Gigante DP, Demarco FF, Peres KG, Peres MA. Association between obesity and periodontal disease in young adults: A population-based birth cohort. J Clin Periodontol 2012;39:717-724.

38. Arora N, Papapanou PN, Rosenbaum M, Jacobs DR Jr., Desvarieux $M$, Demmer RT. Periodontal infection, impaired fasting glucose and impaired glucose tolerance: Results from the Continuous National Health and Nutrition Examination Survey 2009-2010. J Clin Periodontol 2014;41:643-652.

39. Moura-Grec PG, Marsicano JA, Carvalho CA, Sales-Peres SH. Obesity and periodontitis: Systematic review and meta-analysis. Cien Saude Colet 2014;19:1763-1772.

40. Palle AR, Reddy CM, Shankar BS, Gelli V, Sudhakar J, Reddy KK. Association between obesity and chronic periodontitis: A cross-sectional study. J Contemp Dent Pract 2013;14:168-173.

41. Eke PI, Genco RJ. CDC periodontal disease surveillance project: Background, objectives, and progress report. JPeriodontol2007;78(Suppl. 7):S1366-S1371.

Correspondence: Dr. Paul I. Eke, Division of Population Health, National Center for Chronic Disease and Health Promotion, Centers for Disease Control and Prevention, Atlanta, GA 30341. Fax: 770/488-5964; e-mail: peke@ cdc.gov.

Submitted January 7, 2016; accepted for publication May 17, 2016. 